\title{
The Mechanism of the Formation \\ and Identification of Single Radio Frequency \\ Relations Ergotehnicheskih Radio-Electronic Means
}

\author{
Andrey N. Potapov* \\ Military Training and Research Center of the Air Force \\ «Air Force Academy ft. Professor N.E. Zhukovsky and Y.A. Gagarin» \\ 54a Starykh Bol'shevikov Str., Voronezh, 394064, Russia
}

Received 17.10.2015, received in revised form 27.01.2015, accepted 21.02.2016

Examined the identification problem radio frequency $(R F)$ of functioning of radio monitoring of objects for the operational and objectively of identifying such causal RF-of relations between radio electronic facilities (REF) and the medium, by which would have to determine whether a conflict of application REF based on the formation mechanism and identifying of single radio frequency relationship.

Keywords: conflict, a radio frequency, management, radio monitoring, radio-electronic means, model, ergo technical indicator, single.

Citation: Potapov A.N. The mechanism of the formation and identification of single radio frequency relations ergotehnicheskih radio-electronic means, J. Sib. Fed. Univ. Eng. technol., 2016, 9(3), 448-455. DOI: 10.17516/1999-494X-2016-9-3-448-455.

\section{Механизм формирования и идентификации \\ одноместных радиочастотных отношений \\ эрготехнических радиоэлектронных средств}

А.Н. Потапов

ВУНЦ ВВС «ВВА им. проф. Н.Е. Жуковского и Ю.А. Гагарина» Россия, 371600, Воронеж, ул. Старых Большевиков, 54 а

Рассмотрена задача идентификации радиочастотных (РЧ) условий функционирования объектов радиомониторинга для оперативного и объективного выявления таких причинноследственных РЧ-отношений между радиоэлектронными средствами (РЭС) и средой, по которым бы определялся факт возникновения конфликта применения РЭС на основе механизма формирования и идентификачии одноместных радиочастотных отношений.

(C) Siberian Federal University. All rights reserved

* Corresponding author E-mail address: anpotapov36@yandex.ru 
Ключевые слова: конфликт, радиочастотный, управление, радиомониторинг, радиоэлектронное средство, модель, эрготехнический, индикатор, одноместный.

\section{Введение}

Поскольку существующие механизмы радиомониторинга не обеспечивают идентификацию конфликта применения РЭС, возникновение которого является объективной реальностью независимо от отсутствия этих механизмов, то использование метода формирования содержательного компонента учебных упражнений по факту изменения РЧ-условий не всегда целесообразно, а порой расточительно [1].

Для разрешения этой проблемы необходимо выработать метод идентификации РЧ-условий функционирования объектов радиомониторинга, обеспечивающий оперативное и объективное выявление таких причинно-следственных РЧ-отношений между РЭС и средой, по которым бы определялся факт возникновения конфликта применения РЭС и которые можно было бы использовать в качестве входных параметров для моделей РЧ-условий функционирования $M F_{S} \in M_{S Q}$ и $M F_{Q} \in M_{S Q}$ соответственно РЭС $S$ и среды $Q$.

В качестве отправного момента для определения метода идентификации РЧ-условий воспользуемся подходом, базирующимся на преломлении к рассматриваемой предметной области и развитии положений теории системных конфликтов [2]. Несомненным достоинством этого подхода служит структурно-параметрическое представление РЭС, что изначально не только учитывает РЧ-условия, но и определяет предпосылку по индикации конфликта их применения. При этом индикатором сопутствующего признака конфликта применения РЭС может выступать производная от вещественной функции полезности функционирования РЭС по РЧдействию РЭС среды.

Однако из-за того, что отсутствует универсальное описание пространства системного взаимодействия РЭС и полное представление топологии причинно-следственных РЧ-отношений, соответственно, учитывающих различные типы РЧ-связанности между объектами радиомониторинга и относительное поведение их функций полезности, проблематичным остается адекватное описание механизмов формирования РЧ-воздействия, а значит, и идентификация конфликта применения РЭС. В связи с этим рассмотрим ниже эти моменты в указанных аспектах.

\section{Формирование одноместных отношений}

На основании того, что РЧ-воздействия являются причинно-следственными отношениями РЧ-действия и РЧ-влияний, отдельно проанализируем механизмы формирования последних для детализации целостного представления о первом.

Под РЧ-действием РЭС $S_{j}$ будем понимать любое изменение либо его РЧ-входа, либо его РЧ-выхода, приводящее к изменениям процесса функционирования [1].

Задание РЧ-действия $\Delta_{j}=\left\{\Delta_{x j}, \Delta_{y j}\right\}$ РЭС формируется через приращения $\Delta r_{j}=\left\{\Delta x_{j}, \Delta y_{j}\right\}$ значений РЧ-входов и РЧ-выходов, т.е. считается $\Delta_{j} \sim \rho_{j}(t)+\Delta \rho_{j}(t)$, более того $\Delta_{j}=\rho_{j}(t)+\Delta \rho_{j}(t)$ :

$$
\begin{gathered}
\Delta_{x_{j}}=x_{j}(t)+\Delta x_{j}(t)=\left(E_{x_{j}}(t)+\Delta E_{x_{j}}(t)\right) \exp \left\{i\left(\omega_{x_{j}} t+\varphi_{x_{j}}\right)\right\} ; \\
\Delta_{y_{j}}=y_{j}(t)+\Delta y_{j}(t)=\left(E_{y_{j}}(t)+\Delta E_{y_{j}}(t)\right) \exp \left\{i\left(\omega_{y_{j}} t+\varphi_{y_{j}}\right)\right\}, \\
-449-
\end{gathered}
$$


где $\Delta E_{x j}(t)$ и $\Delta E_{y_{j}}(t)$ - приращения напряженности электромагнитных полей.

РЧ-действия $\Delta_{x j}$ и $\Delta_{y_{j}}$ могут быть следствием изменения соответственно чувствительности приемника и мощности передатчика РЭС $S_{j}$.

Все множество действий $\left\{\Delta_{j}\right\} \subset \xi_{j}(t)$ состоит из трех непересекающихся подмножеств $\left\{\Delta_{j}^{+}\right\}$, $\left\{\Delta_{j}^{0}\right\},\left\{\Delta_{j}^{-}\right\}$[2]: если $\Delta_{j}^{+} \subset\left\{\Delta_{j}^{+}\right\}$, то $q_{j}^{\prime}\left(\Delta_{j}^{+}\right)>0$; если $\Delta_{j}^{0} \subset\left\{\Delta_{j}^{0}\right\}$, то $q_{j}^{\prime}\left(\Delta_{j}^{0}\right)=0$; если $\Delta_{j}^{-} \subset\left\{\Delta_{j}^{-}\right\}$, то $q_{j}^{\prime}\left(\Delta_{j}^{-}\right)<0$.

Понятно, что если РЭС функционирует, то это функционирование в каждый момент времени обеспечивается либо $\Delta_{j}^{+}$, либо $\Delta_{j}^{0}$, либо $\Delta_{j}^{-}$:

$$
\left\{\Delta_{j}\right\}=\left\{\Delta_{j}^{+}\right\} \cup\left\{\Delta_{j}^{0}\right\} \cup\left\{\Delta_{j}^{-}\right\} .
$$

Введем ряд определений, характеризующих два $\mathrm{P} Э С S_{j}, S_{l} \in \boldsymbol{S}$ в условиях $\xi_{j}^{0_{k}}(t)$, $\xi_{k}^{d_{j}}(t) \neq \varnothing$.

Определение 1. РЧ-действием $\Delta_{j k}$ объекта $S_{j} \in \boldsymbol{S}$ на объект $S_{k} \in \boldsymbol{S}$ назовем любое РЧ-действие $\Delta_{j} \in \xi_{j}^{0_{k}}(t)$.

Определение 2. РЧ-влиянием $\beta_{j k}$ объекта $S_{j} \in \boldsymbol{S}$ на объект $S_{k} \in \boldsymbol{S}$ назовем любое РЧ-действие $\Delta_{k} \in \xi_{k}^{d_{j}}(t)$ при условии наличия $\Delta_{j} \in \xi_{j}^{0_{k}}(t)\left(\Delta_{k} \in \xi_{k}^{d_{j}}(t) / \Delta_{j} \in \xi_{j}^{0_{k}}(t)\right)$.

Из этого определения следует, что РЧ-влияние $\beta_{j k}$ на объект $S_{k}$ порождается РЧдействием $\Delta_{j k}$ объекта $S_{j}$ и само является РЧ-действием $S_{k}$, распространенным в пространстве РЧ-связанности $\xi_{k}^{d_{j}}(t)$. Этой паре присуще причинно-следственное отношение: $\Delta_{j k}$ является причиной, а $\beta_{j k}-$ следствием $\left(\Delta_{j k} \rightarrow \beta_{j k}\right)$.

Определение 3. РЧ-воздействием $\Pi_{j k}$ объекта $S_{j} \in \boldsymbol{S}$ на объект $S_{k} \in \boldsymbol{S}$ назовем причинноследственное отношение $\left(\Delta_{j k} \rightarrow \beta_{j k}\right)\left(\Pi_{j k} \sim\left(\Delta_{j k} \rightarrow \beta_{j k}\right), \Delta_{j k} \in \xi_{j}^{0_{k}}(t), \beta_{j k} \in \xi_{k}^{d_{j}}(t)\right)$.

Поскольку $\Delta_{j k}$ и $\beta_{j k}-$ РЧ-действия, то их оценка может быть проведена по соответствующим функциям полезности $q_{j}\left(\Delta_{j k}\right), q_{k}\left(\beta_{j k}\right)$, оценивающим эффективность функционирования соответственно $S_{j}$ и $S_{k}$.

Следовательно, как и ранее, можно считать для $\left\{\Delta_{j k}\right\} \subset \xi_{j}^{0_{k}}(t) \exists\left\{\Delta_{j k}\right\}=\left\{\Delta_{j k}^{+}\right\} \cup\left\{\Delta_{j k}^{0}\right\} \cup\left\{\Delta_{j k}^{-}\right\}$; $\left\{\Delta_{i j}^{+}\right\} \Leftrightarrow q_{j}^{\prime}\left(\Delta_{j k}^{+}\right)>0,\left\{\Delta_{j k}^{0}\right\} \Leftrightarrow q_{j}^{\prime}\left(\Delta_{j k}^{0}\right)=0,\left\{\Delta_{j k}^{-}\right\} \Leftrightarrow \Leftrightarrow q_{j}^{\prime}\left(\Delta_{j k}^{-}\right)<0$ и для $\left\{\beta_{j k}\right\} \subset \xi_{k}^{d_{j}}(t) \exists\left\{\beta_{j k}\right\}=\left\{\beta_{j k}^{+}\right\}$ $\cup\left\{\beta_{j k}^{0}\right\} \cup\left\{\beta_{j k}^{-}\right\} ;\left\{\beta_{j k}^{+}\right\} \Leftrightarrow \Leftrightarrow q_{k}^{\prime}\left(\beta_{j k}^{+}\right)>0,\left\{\beta_{j k}^{0}\right\} \Leftrightarrow q_{k}^{\prime}\left(\beta_{j k}^{0}\right)=0,\left\{\beta_{j k}^{-}\right\} \Leftrightarrow q_{k}^{\prime}\left(B_{j k}^{-}\right)<0$, причем для любой пары подмножеств $\left\{\Delta_{j k}^{r}\right\} \cap\left\{\Delta_{j k}^{v}\right\}=\varnothing \forall r, v \sim\{+, 0,-\}, r \neq v$ и $\left\{\beta_{j k}^{h}\right\} \cap\left\{\beta_{j k}^{w}\right\}=\varnothing \forall h, w \sim\{+, 0,-\}$, $h \neq w$.

РЧ-воздействие же имеет векторную оценку и оценивается по двум функциям $-q_{j}\left(\Delta_{j k}\right)$, $q_{k}\left(\beta_{j k}\right)$. Тогда все множество РЧ-воздействий может быть представлено, как

$$
\left\{\Pi_{j k}\right\}=\left\{\Pi_{j k}^{++}\right\} \cup\left\{\Pi_{j k}^{+0}\right\} \cup\left\{\Pi_{j k}^{+-}\right\} \cup\left\{\Pi_{j k}^{0+}\right\} \cup\left\{\Pi_{j k}^{00}\right\} \cup\left\{\pi_{j k}^{0-}\right\} \cup\left\{\Pi_{j k}^{-+}\right\} \cup\left\{\Pi_{j k}^{-0}\right\} \cup\left\{\Pi_{j k}^{--}\right\} .
$$

Здесь, если $\pi_{j k}^{r h} \in\left\{\Pi_{j k}\right\}$, то $\forall r \sim\{+, 0,-\} \Leftrightarrow q_{j}^{\prime}\left(\Delta_{j k}^{+}\right)>0, q_{j}^{\prime}\left(\Delta_{j k}^{0}\right)=0, q_{j}^{\prime}\left(\Delta_{j k}^{-}\right)<0$ и $\forall h \sim\{+, 0,-\} \Leftrightarrow$ $q_{k}^{\prime}\left(\beta_{j k}^{+}\right)>0, q_{k}^{\prime}\left(\beta_{j k}^{0}\right)=0, q_{k}^{\prime}\left(\beta_{j k}^{-}\right)<0$. Причем, как и ранее, для любой пары подмножеств $\left\{\Pi_{j k}^{r h}\right\} \cap\left\{\Pi_{j k}^{v w}\right\}=\varnothing \forall r, v \sim\{+, 0,-\}, r \neq v$ и $\forall h, w \sim\{+, 0,-\}, h \neq w$. 


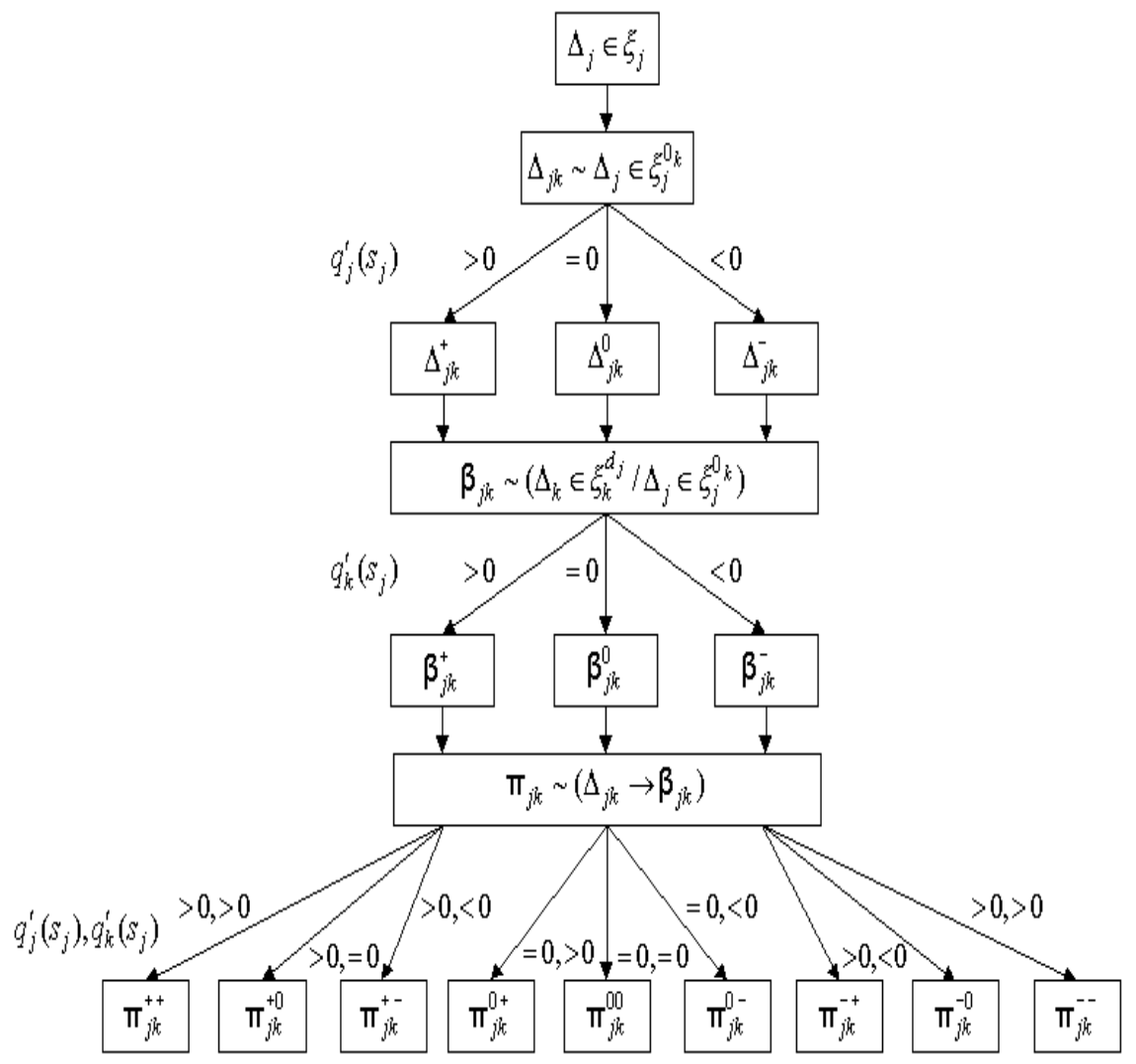

Рис. 1. Формирование введенных отношений - РЧ-действие $\Delta_{k j}$, РЧ-влияние $\beta_{k j}$ и РЧ-воздействие $\Pi_{k j}$

Обратим внимание на то, что введенные отношения - РЧ-действие $\Delta_{k j}$, РЧ-влияние $\beta_{k j}$ и РЧ-воздействие $\pi_{k j}-$ являются односторонними [2]. Они формируются РЧ-действием $\Delta_{j} \mathrm{P} Э О S_{j}$ при условии $\xi_{k}^{d_{j}}(t) \neq \varnothing$ (рис. 1).

Понятно, что если $\xi_{k}^{0_{j}}(t), \xi_{j}^{d_{k}}(t) \neq \varnothing$, то можно построить соответствующие парнонепересекающиеся подмножества:

- РЧ-действий $\left\{\Delta_{k j}^{r}\right\}, \underset{r}{\cup}\left\{\Delta_{k j}^{r}\right\}=\left\{\Delta_{k j}\right\} \forall r \sim\{+, 0,-\} ;$

- РЧ-влияний $\left\{\beta_{k j}^{h}\right\}, \underset{h}{\cup}\left\{\beta_{k j}^{h}\right\}=\left\{\beta_{k j}\right\} \forall h \sim\{+, 0,-\} ;$;

- РЧ-воздействий $\left\{\pi_{k j}^{r h}\right\}, \underset{r}{\cup} \underset{h}{\cup}\left\{\Pi_{k j}^{r h}\right\}=\left\{\Pi_{k j}\right\} \forall r, h \sim\{+, 0,-\}$.

\section{Идентификация одноместных отношений}

С учетом вышеизложенного уместно ввести обобщенный индикатор $J_{j}$ РЧ-воздействия $\Pi_{j k}$ в виде строчной матрицы (вектор-строки) [3]:

$$
J_{j}=\left[\mu_{j}(j), \mu_{j}(k)\right], \mu_{j}(j)=q_{j}^{\prime}\left(\Delta_{j k}\right), \mu_{j}(k)=q_{k}^{\prime}\left(\bigcap_{j k}\right) .
$$

Здесь $\mu_{j}(j) \in \Omega_{j}(j)=\operatorname{Im} q_{j}^{\prime}\left(\Delta_{j k}\right), \mu_{j}(k) \in \Omega_{j}(k)=\operatorname{Im} q_{k}^{\prime}\left(\beta_{j k}\right)$, где $\operatorname{Im} q_{j}^{\prime}, \operatorname{Im} q_{k-}^{\prime}$ образы отображений $q_{j}^{\prime}\left(\Delta_{j k}\right)$ и $q_{k}^{\prime}\left(ß_{j k}\right)$. 
Индикатор $J_{j}$ характеризует результат РЧ-воздействия как с качественной стороны, формируя в некотором смысле «направления» РЧ-воздействий в соответствии с комбинацией знаков $\operatorname{Sign} \mu_{j}(j)$ и $\operatorname{Sign}_{j}(k)(\operatorname{Sign} \omega=-1,0,+1$, при $\omega<0,=0,>0)$, так и с количественной стороны, оценивая в соответствии с величинами $\left|\mu_{j}(j)\right|$ и $\left|\mu_{j}(k)\right|$ силу этих РЧ-воздействий. Множества «направлений» $\forall \operatorname{Sign} \mu_{j}(j)$ и $\forall \operatorname{Sign}_{j}(k)$ образуют в пространстве целевых функций $q_{j}$ и $q_{k}$ не-

который базис РЧ-воздействий $\Lambda_{j k}=\left\{\pi_{j k}^{\left[\operatorname{Sign} \mu_{j}(j), \operatorname{Sign} \mu_{j}(k)\right]}\right\}, \operatorname{Card} \Lambda_{j k}=9$, реализующий базисное множество бинарных отношений [4]:

$$
\begin{aligned}
& \Xi_{j k}=\left\{\mathfrak{R}=\left\{\mathfrak{R}_{\text {inc }}, \mathfrak{R}_{\overline{\text { inc }}_{\text {sim }}}, \mathfrak{R}_{\overline{\text { inc }} \overline{\text { sim }}}\right\} ; \overline{\mathfrak{R}}_{c}=\left\{\overline{\mathfrak{R}}_{\text {cinc }}, \overline{\mathfrak{R}}_{\overline{\text { inc }}_{\text {sim }}}, \overline{\mathfrak{R}}_{\text {cinc }} \overline{\overline{s i m}}\right\} ;\right. \\
& \left.\overline{\mathfrak{R}}_{b}=\left\{\overline{\mathfrak{R}}_{b_{\overline{\text { inc }} \text { sim }}}, \overline{\mathfrak{R}}_{b_{\text {inc }} \overline{\text { sim }}}, \bar{\Re}_{b_{\text {inc }}^{\prime} \overline{\text { sim }}}\right\}\right\} \text {. }
\end{aligned}
$$

Здесь, как и ранее:

- $\mathfrak{R}$ - РЧ-отношение конфликта $\left(q_{k}^{\prime}<0\right): \pi_{j k}^{+-} \Leftrightarrow \mathfrak{R}_{i n c}-$ конфликт в условиях противоречия $\left(q_{j}^{\prime}>0\right), \Pi_{j k}^{0-} \Leftrightarrow \mathfrak{R}_{\overline{\text { inc }} \overline{\text { sim }}}-$ конфликт в условиях непротиворечия и неподобия $\left(q_{j}^{\prime}=0\right)$, $\Pi_{j k}^{--} \Leftrightarrow \mathfrak{R}_{\overline{i n c}_{s i m}}-$ конфликт в условиях непротиворечия и подобия $\left(q_{j}^{\prime}<0\right)$;

- $\overline{\mathfrak{R}}_{c}-$ РЧ-отношение содействия $\left(q_{k}^{\prime}>0\right): \Pi_{j k}^{++} \Leftrightarrow \overline{\mathfrak{R}}_{{ }_{\text {inc }} \overline{s i m}}-$ содействие в условиях непротиворечия и подобия $\left(q_{j}^{\prime}>0\right), \pi_{j k}^{0+} \Leftrightarrow L_{\overline{\text { inc }} \overline{\text { sim }}}-$ содействие в условиях непротиворечия и неподобия $\left(q_{j}^{\prime}=0\right), \pi_{j k}^{-+} \Leftrightarrow \overline{\mathfrak{R}}_{c i n c}-$ содействие в условиях противоречия $\left(q_{j}^{\prime}<0\right)$;

- $\overline{\mathfrak{R}}_{b}-$ РЧ-отношение безразличия $\left(q_{k}^{\prime}=0\right): \pi_{j k}^{+0} \Leftrightarrow \overline{\mathfrak{R}}_{b \overline{\text { inc }}}^{\prime}{ }_{\text {sim }}-$ безразличие в условиях непротиворечия и неподобия $\left(q_{j}^{\prime}>0\right), \pi_{j k}^{00} \Leftrightarrow \overline{\mathfrak{R}}_{b_{\overline{i n c}_{s i m}}}-$ безразличие в условиях непротиворечия и подобия $\left(q_{j}^{\prime}=0\right), \pi_{j k}^{-0} \Leftrightarrow \overline{\mathfrak{R}}_{b \overline{i n c}} \overline{s i m}-$ безразличие в условиях непротиворечия и неподобия $\left(q_{j}^{\prime}<0\right)[5]$.

Заметим, что в частном случае для $\Delta_{j k}$ и $\beta_{j k}$ существуют индикаторы вида $J_{j}^{\Delta}=\left[\mu_{j}(j), 0\right]$ и $J_{j}^{\beta}=\left[0, \mu_{j}(k)\right]$. Формирование $\pi_{j k}^{J_{j}}$ соответствует закону композиции с бинарной операцией $\varphi_{j}\left(\Delta_{j k}, \beta_{j k}\right)=\pi_{j k}^{J_{j}}$ такой, что $\forall \Delta_{j k}, \beta_{j k} \in\left\{\Delta_{j k}\right\} \times\left\{\beta_{j k}\right\}$ существует причинно-следственная связь $\Delta_{j k} \rightarrow \beta_{j k} \sim \Pi_{j k}$ и $J_{j}=J_{j}^{\Delta}+J_{j}^{\beta}$.

Рассмотрим теперь понятие РЧ-взаимовоздействия как совместное выполнение событий РЧ-воздействий $\pi_{j k}^{J_{j}} \wedge \pi_{k j}^{J_{k}}$. Исходя из этого введем РЧ-взаимовоздействие в виде закона компо- 
зиции $\left\{\pi_{j k}^{J_{j}}\right\} \times\left\{\pi_{k j}^{J_{k}}\right\} \rightarrow\left\{\pi_{(j k)}^{J(j k)}\right\} \quad\left(\left\{\pi_{j k}^{J_{j}}\right\}, \quad\left\{\pi_{k j}^{J_{k}}\right\}-\right.$ множества возможных воздействий $S_{j}$ на $S_{k}$ и $S_{k}$ на $S_{j}$ соответственно), который определим как бинарную операцию $\phi_{j k}\left(\pi_{j k}^{J_{j}}, \Pi_{k j}^{J_{k}}\right)=\pi_{(j k)}^{J(j k)}$ по правилу $\forall \Pi_{j k}^{J_{j}}, \Pi_{j k}^{J_{j}} \in\left\{\Pi_{j k}^{J_{j}}\right\} \times\left\{\pi_{k j}^{J_{k}}\right\},\left(\pi_{j k}^{J_{j}} \in\left\{\Pi_{j k}^{J_{j}}\right\}\right) \wedge \wedge\left(\Pi_{k j}^{J_{k}} \in\left\{\Pi_{k j}^{J_{k}}\right\}\right) \Rightarrow \Pi_{(j k)}^{J(j k)} \in\left\{\Pi_{(j k)}^{J(j k)}\right\}$, такой, что индикатор РЧ-взаимовоздействия [6]

$$
J(j k)=J_{j}+J_{k}=\left[\mu_{j k}(j)=\mu_{j}(j)+\mu_{k}(j), \mu_{j k}(k)=\mu_{j}(k)+\mu_{k}(k)\right] .
$$

Иначе $\mu_{j k}(j)=q_{j}^{\prime}\left(\Delta_{j k}\right)+q_{j}^{\prime}\left(\Delta_{j k}\right)$ и $\mu_{j k}(k)=q_{k}^{\prime}\left(ß_{j k}\right)+q_{k}^{\prime}\left(\Omega_{j k}\right)$.

Будем говорить, что $S_{j}$ и $S_{k}$ вступают в бинарное отношение РЧ-воздействия $\left(S_{j} \vec{\pi} S_{k}\right.$ или $\left.\left(S_{j}, S_{k}\right) \in \vec{\pi}\right)$ на множестве $\xi_{j} \times \xi_{j}$, если

$$
\exists \varphi_{j k}: \xi_{j}^{0_{k}}(t) \rightarrow \xi_{k}^{d_{j}}(t) \Leftrightarrow \pi_{j k}^{J_{j}} \in \Lambda_{j k}
$$

Заметим, что определенная таким образом бинарная операция $\varphi_{j k}$ коммутативна, так как

$$
\phi_{j k}\left(\pi{ }_{j k}^{J_{j}}, \Pi_{k j}^{J_{k}}\right)=\Pi_{(j k)}^{J(j k)}=\phi_{k j}\left(\Pi_{k j}^{J_{k}}, \Pi_{j k}^{J_{j}}\right)=\Pi_{(k j)}^{J(k j)} .
$$

Введенное взаимовоздействие $\pi_{(j k)}^{J(j k)}=\Pi_{(k j)}^{J(k j)}=\phi_{j k}\left(\pi_{j k}^{J_{j}}, \Pi_{k j}^{J_{k}}\right)$ полностью отображает наше понимание совместного воздействия $S_{j}$ и $S_{k}[7]$.

Таким образом, РЧ-связанность можно проследить путем построения так называемой матрицы РЧ-достижимости $d=\left[d_{j k}\right]_{N \times N}$, где $d_{j k}=1$, если $Q_{j k} \neq \varnothing ; d_{j k}=0$, если $Q_{j k} \neq \varnothing$.

Отношение $S_{j} \vec{d} S_{k}\left(\vec{d}-\right.$ РЧ-достижимость, если $\left.Q_{j k} \neq \varnothing\right)$ является отображением сюръекции, так как любой элемент из $\xi_{k}^{d_{j}}(t)$ есть образ по крайней мере одного элемента из $\xi_{j}{ }^{0_{k}}(t)$, причем если $\varphi_{j k}: \xi_{j}^{0_{k}}(t) \rightarrow \xi_{k}^{d_{j}}(t)$ и $R_{k}: \xi_{k}^{d_{j}}(t) \rightarrow \xi_{k}(t)$, то их композиция $\left(\varphi_{j k}^{\circ} R_{k}\right): \xi_{j}^{0_{k}}(t) \rightarrow \xi_{k}(t)$, $\left(\varphi_{j k}^{\circ} R_{k}\right) \rho_{j}=\varphi_{j k}\left(R_{k}\left(\rho_{j}\right)\right)$.

С учетом этого композиция отображений $\varphi_{j k}$ и $R_{k}$ позволяет использовать понятие РЧвоздействия $\pi{ }_{j k}^{J} \sim\left(\Delta_{j k} \rightarrow \bigcap_{j k}\right)$ РЭС $S_{j}$ на РЭС $S_{k}$ в виде причинно-следственного отношения $\left(\Delta_{j k} \rightarrow \beta_{j k}\right)$, где $\Delta_{j k}=\left(\rho_{j}(t)+\Delta \rho_{j}(t)\right) \in \xi_{j}^{0_{k}}(t)-$ РЧ-действие $S_{j}$ на $S_{k}, \beta_{j k}=\left(\rho_{j}(t)+\left(\varphi_{j k}\right)^{\prime} \rho_{i} \Delta \rho_{j}(t)\right) \in \xi_{k}^{d_{j}}(t)-$ РЧ-влияние $S_{j}$ на $S_{k}[8]$.

Индикатор $J(j k)$ РЧ-взаимовоздействия позволяет идентифицировать причинноследственные отношения между объектами радиомониторинга для любого типа связанности, когда рассматриваемые множества $\xi_{k}^{d_{j}}(t)$ и $\xi_{j}^{d_{k}}(t)$ являются непустыми и на этих множествах функций полезности $q_{j}$ и $q_{k}$ диагностируемых РЭС $S_{j}$ и $S_{k}$ ведут себя монотонно [9]. В случае, если индикатор $J(j k)$ принимает отрицательные показания, то это означает возникновение конфликта применения РЭС, что служит определяющим фактом по использованию метода формирования конфликтно-устойчивого содержательного компонента учебных упражнений, направленного на выявление полезных предписанных операций для операторов [10]. Очевидно, зная предельные значения $J^{*}(j k)$, определяющие потенциальные возможности РЭС, по величине те- 
кущих отрицательных показаний индикатора $J(j k)$ можно судить о существовании полезных предписанных операций, а значит, о потенциальных способностях применяемого метода.

\section{Заключение}

Множество базисных топологий РЧ-отношений, отражающих существующие типы РЧсвязанности, элементы которого, относящиеся к категории конфликта с точки зрения снижения полезности приминения РЭС из-за изменения РЧ-условий, являются сопутствующими признаками конфликта его применения.

Выработаны механизмы формирования и определены индикаторыпричинно-следственных одноместных РЧ-отношений «объект $\rightarrow$ объект».

\section{Список литературы}

[1] Назаров Т.И., Потапов А.Н. Архитектура структурной конфигурации конфликтноустойчивой автоматизированной системы управления тренажной подготовкой операторов радиоэлектронных объектов: Труды II всероссийской научно-практической конференции «Академические Жуковские чтения» (25-27 ноября 2014 г.). Воронеж: ВУНЦ ВВС «ВВА», 2014. С. 132-135. [Nazarov T.I., Potapov A.N. Architecture structural configuration conflict-resistant trenazhnoy automated control system of radio operator training facilities: Proceedings of II All-Russian scientific-practical conference "Academic Zhukovskiye read" (25-27 November 2014). Voronezh: Military Training and Research Center of the Air Force "Air Force Academy after professor N.E. Zhukovsky and Y. A. Gagarin " 2014, $132-135$ (in Russian)]

[2] Потапов А.Н. Особенности профессиональной подготовки операторов при использовании компьютерных систем тренажа: Сборник статей Всероссийской научно-технической конференции «XXXIV научные чтения, посвященные разработке творческого наследия К.Э.Циолковского», 14-16 сентября 1999 г. Калуга: Калужский дом знаний, 1999.-С.181-184. [Potapov A.N. Features training operators using computer systems Exercises: Collection of papers of All-Russian Scientific and Technical Conference «XXXIV Scientific Conference dedicated to the development of the creative heritage of Tsiolkovsky", September 14-16, 1999. - Kaluga: Kaluga house knowledge, 1999, 181-184 (in Russian)]

[3] Потапов А.Н. Автоматизация тренажной подготовки операторов радиоэлектронных объектов управления воздушным движением, ISBN 978-5-903100-98-9. - Воронеж: Изд-во ВАИУ,2010. - 136 с. [Potapov A.N. Automation trenazhnoy training of operators of radio electronic facilities of air traffic control, ISBN 978-5-903100-98-9. - Voronezh: Publishing house Voronezh Aviation Engineering Institute, 2010, 136 (in Russian)]

[4] Потапов А.Н. Методы и модели повышения эффективности эрготехнических компьютерных систем тренажа на основе оценки их конфликтно-устойчивости. Воронеж: Изд-во ВАИУ,2011. -96 c. [Potapov A.N. Methods and models improve the efficiency of agro-technical computer systems Exercises based on an assessment of their conflict-sustainability. Voronezh: Publishing house Voronezh Aviation Engineering Institute. 2011. 96 (in Russian)]

[5] Потапов А.Н. К вопросу разрешения конфликтов в практической подготовке лиц группы руководства полетами: Материалы I Всероссийской научно-технической конференции «Теория конфликта и ее приложение», 26-29 июня 2000. Воронеж: ВГТА, 2000. С. 24-27. [Potapov 
$A . N$. On the issue of conflict resolution in the practical training of a group of persons flight manual: Materials of I All-Russia scientific-technical conference "Conflict theory and its applications", June 26-29, 2000. Voronezh: VGTA. 2000. 24-27 (in Russian)]

[6] Назаров Т.И., Потапов А.Н. Вестник Тамбовского университета. 2012. Т. 17. Вып. 6. С. 1603-1608. [Nazarov T.I., Potapov A.N. Journal of the University of Tambov. 2012. Volume 17. Issue 6. 1603-1608 (in Russian)]

[7] Потапов А.Н. Вестник Воронежского государственного технического университета. 2013. T.1. №4. C. 22-25. [Potapov A.N. Journal of Voronezh State Technical University. 2013. Volume 1. №4. 22-25 (in Russian)]

[8] Потапов А.Н., Овчаров В.В., Попов Ю.М., Удодов Ю.В. Приборы и системы. Управление, контроль, диагностика. 2012. №7 C.74-79. [Potapov A.N., Ovcharov V.V., Popov Ju.M., Udodov Ju.B. Pribory i sistemy. Upravlenie, kontrol, diagnostika.. 2012. №7. 74-79 (in Russian)]

[9] Алексеев В.В., Потапов А.Н. Вестник Воронежского института МВД России . 2012. №2 C.19-30. [Alexeev V.V., Potapov A.N. Journal of Voronezh Institute of Russian Ministry of Internal Affairs. 2012. №2. 19-30 (in Russian)]

[10] Потапов А.Н.. Сопутствующие признаки функционирования информационных эрготехнических радиоэлектронных систем: Материалы 14 международной научно-методической конференции «Информатика: проблемы, методология, технологии», Т. 1. 6-8 февраля 2014 г. Воронеж: ВГУ 556 с. С. 486-491. [Potapov A.N. Associated signs functioning of information ergotehnicheskih electronic systems: Proceedings of the 14th International Scientific Conference "Information Science: Problems, Methodology, Technology" Volume 1 February 6-8 2014g. Voronezh: Voronezh State University. 486-491 (in Russian)] 